\title{
CONNECTED ESCAPING SETS OF EXPONENTIAL MAPS
}

\author{
Lasse Rempe \\ University of Liverpool, Department of Mathematical Sciences \\ Liverpool L69 7ZL, United Kingdom; l.rempe@liverpool.ac.uk
}

\begin{abstract}
We show that, for many parameters $a \in \mathbf{C}$, the set $I\left(f_{a}\right)$ of points that converge to infinity under iteration of the exponential map $f_{a}(z)=e^{z}+a$ is connected. This includes all parameters for which the singular value $a$ escapes to infinity under iteration of $f_{a}$.
\end{abstract}

\section{Introduction}

If $f: \mathbf{C} \rightarrow \mathbf{C}$ is a transcendental entire function (that is, a non-polynomial holomorphic self-map of the complex plane), the escaping set of $f$ is defined as

$$
I(f):=\left\{z \in \mathbf{C}: f^{n}(z) \rightarrow \infty\right\} .
$$

(Here $f^{n}=\underbrace{f \circ \cdots \circ f}_{n \text { times }}$ denotes the $n$-th iterate of $f$, as usual.)

This set has recently received much attention in the study of transcendental dynamics, due to the structure it provides to the dynamical plane of such functions. It is neither an open nor a closed subset of the complex plane and tends to have interesting topological properties. In the simplest cases (see [DK, B, R $\left.{ }^{3} \mathrm{~S}, \mathrm{R} 4\right]$ ), the set $I(f)$ is homeomorphic to a subset of a "Cantor Bouquet" (a certain uncountable disjoint union of curves to $\infty$ ), and in particular $I(f)$ is disconnected for these functions. It has recently come to light that there are many situations where $I(f)$ is in fact connected. Rippon and Stallard showed that this is the case for any entire function having a multiply-connected wandering domain [RS1] and also for many entire functions of small order of growth [RS2]. These examples have infinitely many critical values. The latter condition is not necessary, as there are even examples of connected escaping sets in the family

$$
f_{a}: \mathbf{C} \rightarrow \mathbf{C} ; \quad z \mapsto \exp (z)+a
$$

of exponential maps, which may be considered the simplest parameter space of transcendental entire functions. (These maps have no critical points, and exactly one asymptotic value, namely the omitted value a.) Indeed, it was shown in [R5] that the escaping set is connected for the standard exponential map $f_{0}$, while all pathconnected components of $I\left(f_{0}\right)$ are relatively closed and nowhere dense. The proof uses previously known results about this particular function, thus leaving open the possibility of the connectedness of $I\left(f_{0}\right)$ being a rather unusual phenomenon.

Motivated by this result, Jarque [J] showed that $I\left(f_{a}\right)$ is connected whenever $a$ is a Misiurewicz parameter, i.e., when the singular value $a$ is preperiodic. In this

doi:10.5186/aasfm.2011.3604

2010 Mathematics Subject Classification: 37F10, 30D05.

Key words: Julia set, exponential map, Eremenko's Conjecture.

Supported by EPSRC Grant EP/E017886/1 and Fellowship EP/E052851/1. 
note, we extend his proof to a wider class of parameters. Our results suggest that connectedness of the escaping set is in fact true for "most" parameters for which the singular value belongs to the Julia set $J\left(f_{a}\right)=\overline{I\left(f_{a}\right)}$. ${ }^{1}$ If $a \notin J\left(f_{a}\right)$, then $f_{a}$ has an attracting or parabolic periodic orbit, and it is well-known that the Julia set, and hence the escaping set, is a disconnected subset of $\mathbf{C}$.

The main condition used in our paper is the following combinatorial notion, first introduced in [R3].

1.1. Definition. We say that the singular value $a$ of an exponential map $f=f_{a}$ is accessible if $a \in J(f)$ and there is an injective curve $\gamma:[0, \infty) \rightarrow J(f)$ with $\gamma(0)=a, \gamma(t) \in I(f)$ for $t>0$ and $\operatorname{Re} \gamma(t) \rightarrow \infty$ as $t \rightarrow \infty$.

Remark 1. It follows from [FRS, Corollary 4.3] that this definition is indeed equivalent to the one given in [R3]. In particular, the requirement $\operatorname{Re} \gamma(t) \rightarrow \infty$ could be omitted.

Remark 2. It is not known whether the condition that the singular value $a$ is accessible is always satisfied when $a$ belongs to the Julia set (as far as we know, this is an open question even for quadratic polynomials). Known cases include all Misiurewicz parameters, all parameters for which the singular value escapes and a number of others. Compare [R3, Remark 2 after Definition 2.2].

If $\gamma$ is as in this definition, then every component of $f^{-1}(\gamma)$ is a curve tending to $\infty$ in both directions. The set $\mathbf{C} \backslash f^{-1}(\gamma)$ consists of countably many "strips" $S_{k}$ $(k \in \mathbf{Z})$, which we will assume are labelled such that $S_{k}=S_{0}+2 \pi i k$ for all $k$. For our purposes, it does not matter which strip is labelled as $S_{0}$, although it is customary to use one of two conventions: either $S_{0}$ is the strip that contains the points $r+\pi i$ for sufficiently large $r$, or alternatively the strip containing the singular value $a$ (provided that $f(a) \notin \gamma)$.

For any point $z \in \mathbf{C} \backslash I(f)$, there is a sequence $\underline{\mathrm{u}}=\mathrm{u}_{0} \mathrm{u}_{1} \mathrm{u}_{2} \ldots$ of integers, called the itinerary with respect to this partition, such that $f^{j}(z) \in S_{\mathbf{u}_{j}}$ for all $j \geq 0$. Every escaping point whose orbit does not intersect the curve $\gamma$ also has such an itinerary. The itinerary of the singular value (if it exists) is called the kneading sequence of $f$.

1.2. Theorem. Let $f(z)=\exp (z)+a$ be an exponential map. If

(a) the singular value a belongs to the escaping set, or

(b) the singular value a belongs to $J(f) \backslash I(f)$ and is accessible with non-periodic kneading sequence,

then $I(f)$ is a connected subset of $\mathbf{C}$.

Remark 1. All path-connected component of $I(f)$ are nowhere dense under the hypotheses of the theorem [R3, Lemma 4.2].

Remark 2. The theorem applies, in particular, to the exponential map $f=\exp$; this gives an alternative proof of the main result of [R5].

Conjecturally, if $f_{a}$ has a Siegel disk with bounded-type rotation number, then the singular value $a$ is accessible in our sense, and furthermore accessible from the Siegel disk. In this case, the kneading sequence would be periodic and the Julia set (and hence the escaping set) disconnected. On the other hand, it is plausible that

\footnotetext{
${ }^{1}$ The Julia set of $f$ is defined as the set of non-normality of the family of iterates of $f$. For certain entire functions, including all exponential maps, it coincides with the closure of $I(f)$ by [E, EL].
} 
the escaping set of $f_{a}$ is connected whenever $f_{a}$ does not have a nonrepelling periodic orbit.

The second half of Theorem 1.2 does not have a straightforward generalization to other families. Indeed, our proof relies on the fact that $f^{-1}(\gamma) \subset I(f)$ (because the singular value $a$ is omitted), and that connected sets of nonescaping points therefore cannot cross the partition boundaries. In fact, Mihaljević-Brandt [M-B] has shown that for many postcritically preperiodic entire functions, including those in the complex cosine family $z \mapsto a \exp (z)+b \exp (-z)$, the escaping set is disconnected. On the other hand, the proof of the first part of our theorem should apply to much more general functions; in particular, to all cosine maps for which both critical values escape.

We recall that Eremenko's conjecture [E] states that every connected component of the escaping set of a transcendental entire function is unbounded. This is true for all exponential maps [SZ] and indeed for much larger classes of entire functions $\left[\mathrm{R}^{3} \mathrm{~S}, \mathrm{R} 2\right]$. Despite progress, the question remains open in general, while it is now known that some related but stronger properties may fail (compare, e.g., $\left[\mathrm{R}^{3} \mathrm{~S}\right]$ ). The connectivity of the escaping set for a wide variety of exponential maps illustrates some of the counterintuitive properties one may encounter in the study of connected components of a planar set that is neither open nor closed (and exposes the difficulties of constructing a counterexample should the conjecture turn out to be false). It seems likely that a better understanding of these phenomena will provide further insights into Eremenko's conjecture.

Structure of the article. In Section 2, we collect some background about the escaping set of an exponential map. In Section 3, we establish an important preliminary result. The proof of Theorem 1.2 is then carried out in Section 4, separated into two different cases (Theorems 4.3 and 4.4).

Basic notation. As usual, we denote the complex plane by $\mathbf{C}$, and the Riemann sphere by $\hat{\mathbf{C}}=\mathbf{C} \cup\{\infty\}$. The closure of a set $A$ in $\mathbf{C}$ and in $\hat{\mathbf{C}}$ will be $\operatorname{denoted} \bar{A}$, resp. $\hat{A}$. Boundaries will be understood to be taken in $\hat{\mathbf{C}}$, unless explicitly stated otherwise.

Acknowledgments. I would like to thank Xavier Jarque, Phil Rippon and Gwyneth Stallard for interesting discussions, and the referee for helpful comments.

\section{Escaping points of exponential maps}

It was shown by Schleicher and Zimmer [SZ] that the escaping set $I\left(f_{a}\right)$ of any exponential map is organized in curves to infinity, called dynamic rays or hairs, which come equipped with a combinatorial structure and ordering. We do not require a precise understanding of this structure. Instead, we take an axiomatic approach, collecting here only those properties that will be used in our proofs.

2.1. Proposition. Let $f(z)=\exp (z)+a$ be an exponential map.

(a) If $a \in I(f)$, then $a$ is accessible in the sense of Definition 1.1.

(b) Suppose that $U \subset \mathbf{C}$ is an open set with $U \cap J(f) \neq \emptyset$. Then there is a curve $\gamma:[0, \infty) \rightarrow I(f)$ with $\gamma(0) \in U$ and $\operatorname{Re} \gamma(t) \rightarrow \infty$ as $t \rightarrow \infty$.

Proof. The first statement follows from [SZ, Theorem 6.5]. 
To prove the second claim, we use the fact that there is a collection of uncountably many pairwise disjoint curves to $\infty$ in the escaping set. (This also follows from [SZ], but has been known much longer: see [DGH, DT].)

Hence there is a curve $\alpha:[0, \infty) \rightarrow I(f)$ with $\lim _{t \rightarrow \infty}|\alpha(t)|=\infty$ and $f^{j}(a) \notin \alpha$ for all $j \geq 0$. In particular, $f^{-1}(\alpha(0))$ is an infinite set, and by Montel's theorem, there exist $n \geq 1$ and some $z_{0} \in U$ such that $f^{n}\left(z_{0}\right)=\alpha(0)$. We can analytically continue the branch of $f^{-n}$ that takes $\alpha(0)$ to $z_{0}$ to obtain a curve $\gamma:[0, \infty) \rightarrow I(f)$ with $f^{n} \circ \gamma=\alpha$ and $\gamma(0)=z_{0} \in U$.

We have $|f(\gamma(t))| \rightarrow \infty$ as $t \rightarrow \infty$. As $|f(z)| \leq \exp (\operatorname{Re}(z))+|a|$ for all $z$, we thus have $\operatorname{Re} \gamma(t) \rightarrow+\infty$ as $t \rightarrow \infty$, as claimed.

Exponentially bounded itineraries. In this subsection, we fix an exponential map $f(z)=\exp (z)+a$ with accessible singular value, and an associated partition into itinerary strips $S_{j}$. Recall that we defined the itinerary of a point only if its orbit never belongs to the strip boundaries.

It simplifies terminology if we can speak of itineraries for all points. Hence we adopt the (slightly non-standard) convention that any sequence $\underline{u}=u_{0} u_{1} u_{2} \ldots$ with $f^{j}(z) \in \overline{S_{\mathrm{u}_{j}}}$ is called an itinerary of $z$. Thus $z$ has a unique itinerary if and only if its orbit does not enter the strip boundaries.

2.2. Definition. An itinerary $\underline{u}=u_{0} u_{1} u_{2} \ldots$ is exponentially bounded if there is a number $x \geq 0$ such that $2 \pi\left|\mathrm{u}_{j}\right| \leq \exp ^{j}(x)$ for all $j \geq 0$.

Remark 1. At first glance it may seem that the itinerary of every point $z \in \mathbf{C}$ is exponentially bounded, since certainly $\left|f^{n}(z)\right|$, and thus $\left|\operatorname{Im} f^{n}(z)\right|$, are exponentially bounded sequences. However, in general, we have no a priori control over how the imaginary parts in the strips $S_{j}$ behave as the real parts tend to $-\infty$.

Nonetheless, it seems plausible that all points have exponentially bounded itineraries; certainly this is true for well-controlled cases such as Misiurewicz parameters. We leave this question aside, as its resolution is not required for our purposes.

Remark 2. If $z$ does not have a unique itinerary, we take the statement " $z$ has exponentially bounded itinerary" to mean that all itineraries of $z$ are exponentially bounded. However, two itineraries of $z$ differ by at most 1 in every entry, so this is equivalent to saying that $z$ has at least one exponentially bounded itinerary.

2.3. Proposition. If $z \in \mathbf{C}$ belongs to the closure of some path-connected component of $I(f)$ (in particular, if $z \in I(f)$ or $z=a$ ), then $z$ has exponentially bounded itinerary.

Proof. Let $z_{0} \in I(f)$, and let $\underline{u}$ be an itinerary of $z_{0}$. Then $\operatorname{Re} f^{j}\left(z_{0}\right) \rightarrow+\infty$, and in particular there exists $R \in \overline{\mathbf{R}}$ such that $\operatorname{Re} f^{n}\left(z_{0}\right) \geq R$ for all $j \geq 0$.

The domain $S_{0}$ is bounded by two components of $f^{-1}(\gamma)$. Each of these has bounded imaginary parts in the direction where the real parts tend to $+\infty$. (In fact, each preimage component is asymptotic to a straight line $\{\operatorname{Im} z=2 \pi k\}$ for some $k \in \mathbf{Z}$, but we do not require this fact.) In particular,

$$
M:=\sup \left\{|\operatorname{Im} z|: z \in \overline{S_{0}}, \operatorname{Re} z \geq R\right\}<\infty .
$$

Then it follows that $\left|\operatorname{Im} f^{j}\left(z_{0}\right)-2 \pi u_{j}\right| \leq M$ for all $j$, and hence

$$
2 \pi\left|\mathbf{u}_{j}\right| \leq\left|\operatorname{Im} f^{j}\left(z_{0}\right)\right|+M
$$


Set $\alpha:=\ln (3(|a|+M+2))$. Elementary calculations give

$$
\begin{aligned}
\exp (|z|+\alpha) & =3(|a|+M+2) \exp (|z|) \geq \exp (|z|)+2(|a|+M+2) \\
& \geq \exp (\operatorname{Re} z)+|a|+M+\ln 3+(|a|+M+2) \\
& \geq \exp (\operatorname{Re} z)+|a|+M+\alpha \geq|f(z)|+M+\alpha
\end{aligned}
$$

for all $z \in \mathbf{C}$. It follows that

$$
2 \pi\left|\mathrm{u}_{j}\right| \leq\left|\operatorname{Im} f^{j}\left(z_{0}\right)\right|+M \leq\left|f^{j}\left(z_{0}\right)\right|+M \leq \exp ^{j}\left(\left|z_{0}\right|+\alpha\right)
$$

for all $j \geq 0$, so $z_{0}$ has exponentially bounded itinerary.

Also, it is shown in [FRS] that the partition boundaries, i.e., the components of $f^{-1}(\gamma)$, are path-connected components of $I(f)$ (where $\gamma \subset I(f)$ is the curve connecting the singular value to infinity). So if $C$ is the path-connected component of $I(f)$ containing $z_{0}$, then $f^{j}(C) \subset \overline{S_{\mathbf{u}_{j}}}$, and hence $f^{j}(\bar{C}) \subset \overline{S_{\mathbf{u}_{j}}}$, for all $j \geq 0$. So all points in $\bar{C}$ have exponentially bounded itinerary, as claimed.

Escaping endpoints. There are two types of escaping points:

2.4. Definition. Suppose that $f_{a}$ is an exponential map and $z \in I(f)$. We say that $z$ is a non-endpoint if there is an injective curve $\gamma:[-1,1] \rightarrow I\left(f_{a}\right)$ with $\gamma(0)=z$; otherwise $z$ is called an endpoint.

It follows from [FRS] that this coincides with the classification into "escaping endpoints of rays" and "points on rays" given in [SZ]; we use the above definition here because it is easier to state. In [SZ], escaping endpoints were completely classified; we only require the following fact.

2.5. Proposition ([SZ]). Let $f_{a}$ be an exponential map with $a \in I(f)$ (so in particular $a$ is accessible), and suppose $a$ is an endpoint. Then the kneading sequence of $f$, i.e., the itinerary of $a$, is unique and unbounded.

For exponential maps with an attracting fixed point, any non-endpoint is inaccessible from the attracting basin [DG]. The following is a variant of this fact that holds for every exponential map.

2.6. Proposition. Suppose that $f(z)=\exp (z)+a$ is an exponential map and suppose that $z \in I(f)$ is not an endpoint. Then any closed connected set $A \subset \mathbf{C}$ with $z \in A$ and $\# A>1$ contains uncountably many escaping points.

Sketch of proof. The idea is that any path-connected component of $I(f)$ is accumulated on both from above and below by other such components. This is by now a well-known argument; see, e.g., [R3, Lemma 3.3] and [ReS, Lemma 13], where it is used in a slightly different context. We provide a few more details for completeness.

We may assume that $A$ intersects only countably many different path-connected components of $I(f)$; otherwise we are done. Let $\gamma:[-1,1] \rightarrow I(f)$ be as in Definition 2.4, with $\gamma(0)=z$.

Then there are two sequences $\gamma_{n}^{+}:[-1,1] \rightarrow I(f)$ and $\gamma_{n}^{-}:[-1,1] \rightarrow I(f)$ of curves that do not intersect $A$ and that converge locally uniformly to $\gamma$ from both sides of $\gamma$. Since $A$ is closed, it follows that we must have either $\gamma([-1,0]) \subset A$ or $\gamma([0,1]) \subset A$.

The same argument yields the following result. 
2.7. Proposition. (Accumulation on a) Suppose that $f=\exp (z)+a$ is an exponential map with accessible singular value, and let $\gamma \subset I(f)$ be a curve as in the definition of accessibility.

If $A \subset \mathbf{C} \backslash I(f)$ is a connected set with $\bar{A} \cap \gamma \neq \emptyset$, then $a \in \bar{A}$.

Sketch of proof. As in the above proof, there are two sequences of curves that converge to $\gamma$ from both sides. (See also [R1, Lemmas 4.7 and 5.1].) In order to accumulate on any point of $\gamma$, the set $A$ will need to pass arbitrarily close to the endpoint $a$ of the curve.

\section{Closed subsets of non-escaping points}

Let us say that a set $A \subset \mathbf{C}$ disconnects the set $C \subset \mathbf{C}$ if $C \cap A=\emptyset$ and (at least) two different connected components of $\mathbf{C} \backslash A$ intersect $C$. The following lemma was used in $[\mathrm{J}]$ to prove the connectivity of the escaping set for Misiurewicz exponential maps.

3.1. Lemma. Let $C \subset \mathbf{C}$. Then $C$ is disconnected if and only if there is a closed connected set $A \subset \mathbf{C}$ that disconnects $C$.

Proof. The "if" part is trivial. If $C$ is disconnected, then by the definition of connectivity there are two points $z, w \in C$ and an open set $U \subset \mathbf{C}$ with $\partial U \cap C=\emptyset$ such that $z \in U$ and $w \notin U$. By passing to a connected component if necessary, we may assume that $U$ is connected. Let $V$ be the connected component of $\hat{\mathbf{C}} \backslash \hat{U}$ that contains $w$. Then $V$ is simply connected with $\partial V \subset \partial U \subset \hat{\mathbf{C}} \backslash C$. It follows that $\mathrm{C} \backslash V$ has exactly one connected component (since every such connected component intersects the connected set $\bar{U})$. Thus $A:=\partial V \cap \mathbf{C}$ is a closed connected set that disconnects $z$ and $w$, as required.

Thus, in order to prove the connectedness of the escaping set, we need to study closed connected sets of non-escaping points and show that these cannot disconnect $I(f)$. The following proposition will be the main ingredient in this argument.

3.2. Proposition. Let $f=f_{a}$ be an exponential map with accessible singular value $a$. Let $A \subset \mathbf{C}$ be closed and connected. Suppose that furthermore the points in $A$ have uniformly exponentially bounded itineraries, i.e., there exists a number $x$ with the following property: if $n \geq 0$ and $\mathrm{u} \in \mathbf{Z}$ such that $f^{n}(A) \cap \overline{S_{\mathrm{u}}} \neq \emptyset$, then $2 \pi|\mathrm{u}| \leq \exp ^{n}(x)$.

If $A \cap I(f)$ is bounded, then there is $n \geq 0$ such that $f^{n}(A)$ is bounded.

This is essentially a (simpler) variant of [R3, Lemma 6.5], and can be proved easily in the same manner using the combinatorial terminology of that paper. Instead, we give an alternative proof - quite similar to the proof of the main theorem of $[\mathrm{J}]$ - that does not require familiarity with these concepts.

Proof. We prove the converse, so suppose that $f^{n}(A)$ is unbounded for all $n$. (In particular, $A$ is nonempty.) We need to show that $A \cap I(f)$ is unbounded.

Similarly as in the proof of Proposition 2.3, set

$$
M:=\sup \left\{|\operatorname{Im} z|: z \in S_{0}, \operatorname{Re} z \geq 0\right\}
$$


and $\alpha:=\ln (3(|a|+M+2))$. Also pick some $z_{0} \in A$ and let $x_{0} \geq \max \left(\left|z_{0}\right|, x\right)+\alpha$ be arbitrary. The hypotheses and (2.1) imply that

$$
\left|\operatorname{Im} f^{n}(z)\right| \leq \exp ^{n}(x)+M \leq \exp ^{n}\left(x_{0}\right)
$$

whenever $z \in A$ and $n \geq 0$ such that $\operatorname{Re} f^{n}(z) \geq 0$. Also, again by (2.1),

$$
\left|f^{n}\left(z_{0}\right)\right| \leq \exp ^{n}\left(x_{0}\right) .
$$

Let $n \in \mathbf{N}$. Recall that $f^{n}(A)$ is connected and unbounded by assumption. Hence by (3.2), there exists some $z_{n} \in A$ with

$$
\left|f^{n}\left(z_{n}\right)\right|=\exp ^{n}\left(x_{0}\right) .
$$

We claim that

$$
\exp ^{j}\left(x_{0}\right)-1 \leq\left|f^{j}\left(z_{n}\right)\right| \leq 2 \exp ^{j}\left(x_{0}\right)+1
$$

for $j=0, \ldots, n$. Indeed, if $j<n$ is such that (3.3) is true for $j+1$, then

$$
\begin{aligned}
\operatorname{Re} f^{j}\left(z_{n}\right) & =\ln \left|f^{j+1}\left(z_{n}\right)-a\right| \geq \ln \left(\left|f^{j+1}\left(z_{n}\right)\right|-|a|\right) \\
& \geq \ln \left(\exp ^{j+1}\left(x_{0}\right)-|a|-1\right)=\exp ^{j}\left(x_{0}\right)-\ln \frac{\exp ^{j+1}\left(x_{0}\right)}{\exp ^{j+1}\left(x_{0}\right)-|a|-1} \\
& \geq \exp ^{j}\left(x_{0}\right)-\ln 2>\exp ^{j}\left(x_{0}\right)-1 .
\end{aligned}
$$

Similarly, we see that

$$
\operatorname{Re} f^{j}\left(z_{n}\right) \leq \exp ^{j}\left(x_{0}\right)+1 \text {. }
$$

Together with (3.1), this yields (3.3) for $j$.

Now let $z$ be any accumulation point of the sequence $z_{n}$; since $A$ is closed (and the sequence is bounded), we have $z \in A$. By continuity, (3.3) holds also for $z$, and hence $z \in A \cap I(f)$. As $x_{0}$ can be chosen arbitrarily large, we have shown that $A \cap I(f)$ is unbounded, as required.

\section{Proof of Theorem 1.2}

The following two lemmas study the properties of sets that can disconnect the escaping set of an exponential map with accessible singular value.

4.1. Lemma. Let $f$ be an exponential map and suppose that $A \subset \mathbf{C} \backslash I(f)$ disconnects the escaping set. Then $A$ contains points with arbitrarily large real parts.

Proof. This follows immediately from Proposition 2.1 (b).

4.2. Lemma. Let $f=f_{a}$ be an exponential map with accessible singular value a. Suppose that $A \subset \mathbf{C} \backslash I(f)$ is connected and disconnects the escaping set. Then

(a) If the real parts of the points of $A$ are bounded from below, then $f(A)$ also disconnects the escaping set.

(b) The common itinerary of the points of $A$ is exponentially bounded.

Proof. Let $\gamma$ be the curve from Definition 1.1. Let $U$ be the component of $\mathbf{C} \backslash A$ that contains a left half plane, and let $V \neq U$ be another component of $\mathbf{C} \backslash A$ with $V \cap I(f) \neq \emptyset$. (Such a component exists by assumption.) Every component of $f^{-1}(\gamma)$ intersects every left half plane. Thus $f^{-1}(\gamma) \subset U$, and in particular $\bar{V} \cap f^{-1}(\gamma)=\emptyset$. 
This means that $\bar{V}$ is contained in a single itinerary domain $S_{j}$ and the real parts of the points of $V$ are bounded from below. Since $\bar{V}$ intersects $A$, it follows that $A$ is contained in the same itinerary domain $S_{j}$. (Recall that $A$ is connected and does not intersect the escaping set.) As $\left.f\right|_{S_{j}}$ is a conformal isomorphism between $S_{j}$ and $\mathbf{C} \backslash \gamma$, it follows that $f(V)$ is a component of $\mathbf{C} \backslash(f(A) \cup \gamma)$. Now $f(A)$ does not accumulate on the singular value $a$ by assumption, and it follows from Proposition 2.7 that $\overline{f(A)}$ does not intersect $\gamma$. It follows that $f(V)$ is a connected component of $\mathbf{C} \backslash f(A)$ that intersects the escaping set but does not intersect $\gamma$. Hence $f(A)$ disconnects $I(f)$, which proves the first claim.

Note that the second claim is trivial if $\bar{A}$ intersects the escaping set, since every escaping point has exponentially bounded itinerary by Proposition 2.3. So we may suppose that $A$ is a closed set. Also recall that $a$ has exponentially bounded itinerary, which means that we may assume that $a \notin \overline{f^{n}(A)}$ for all $n \geq 0$. Then the real parts of the points of $f^{n}(A)$ are bounded from below for all $n$.

Hence for all $n \geq 0, f^{n}(A)$ is a closed subset of $\mathbf{C}$ with real parts bounded from below and disconnecting $I(f)$. Let us say that $f^{n}(A)$ surrounds a set $X \subset \mathbf{C} \backslash f^{n}(A)$ if $X$ does not belong to the component of $\mathbf{C} \backslash f^{n}(A)$ that contains a left half plane.

By assumption, $A$ surrounds some escaping point $z_{0}$. We claim that, for every $n \geq 0$,

$(*) f^{n}(A)$ surrounds either $f^{n}\left(z_{0}\right)$ or $f^{j}(a)$ for some $j<n$.

This follows by induction using an argument similarly as in the first part of the proof. Indeed, let $w=f^{n}\left(z_{0}\right)$ or $w=f^{j}(a)$ be the point surrounded by $f^{n}(A)$ by the induction hypothesis, let $U$ be the component of $\mathbf{C} \backslash f^{n}(A)$ containing $w$, and let $S_{j}$ be the itinerary strip containing $f^{n}(A)$ and hence $U$. Now $f: S_{j} \rightarrow \mathbf{C} \backslash \gamma$ is a conformal isomorphism. Thus either $f(U)$, and hence $f(w)$, is surrounded by $f^{n+1}(A)$, or $U$ is mapped to the component of $\mathbf{C} \backslash f^{n+1}(A)$ that contains a left half plane and $f^{n+1}(A)$ surrounds $\gamma$, and hence $a$. The induction is complete in either case.

Because $z_{0}$ and $a$ both have exponentially bounded itineraries, it follows from $\left({ }^{*}\right)$ that all points in $A$ do also.

Now we are ready to prove Theorem 1.2. We begin by treating the case where $f$ has a unique and non-periodic kneading sequence. This includes the second case of Theorem 1.2, as well as the case of all escaping endpoints.

4.3. Theorem. Suppose that $f=f_{a}$ is an exponential map with accessible singular value with unique kneading sequence $\underline{\mathrm{u}}=\mathrm{u}_{0} \mathrm{u}_{1} \mathrm{u}_{2} \ldots$ If $\underline{\mathrm{u}}$ is not periodic, then $I(f)$ is connected.

Proof. We prove the converse. So suppose that $I(f)$ is disconnected; we must show that $\underline{\mathrm{u}}$ is periodic. By Lemma 3.1, there exists a closed connected set $A \subset \mathbf{C}$ that disconnects the set of escaping points. Then all points of $A$ have a common itinerary $\underline{\underline{u}}^{\prime}$, and this itinerary is exponentially bounded by Lemma 4.2. Note that

(*) If $k \geq 0$ is such that $f^{k}(A)$ is unbounded to the left, then $a \in \overline{f^{k+1}(A)}$, and hence $\sigma^{k+1}\left(\underline{\mathrm{u}}^{\prime}\right)=\underline{\mathrm{u}}$.

(Here $\sigma$ denotes the shift map; i.e., $\sigma\left(\mathrm{u}_{0} \mathrm{u}_{1} \mathrm{u}_{2} \ldots\right)=\mathrm{u}_{1} \mathrm{u}_{2} \ldots$ )

By Proposition 3.2, $f^{k}(A)$ is bounded for some $k$; let $k_{1}$ be minimal with this property. Since $A$ is unbounded by Lemma 4.1, we must have $k_{1}>0$, and since 
$f^{k_{1}-1}(A)$ is contained in one of the domains $S_{j}$, it follows that $f^{k_{1}-1}(A)$ is unbounded to the left.

Now let $k_{0}$ be the minimal number for which $f^{k_{0}}(A)$ is unbounded to the left. By Lemma 4.2, $f^{k_{0}}(A)$ also disconnects the escaping set, and hence is unbounded to the right by Lemma 4.1. Thus $f^{k_{0}+1}(A)$ is unbounded, and therefore $k_{0}+1<k_{1}$ by definition.

So $(*)$ implies that

$$
\sigma^{k_{1}}\left(\underline{\mathrm{u}}^{\prime}\right)=\underline{\mathrm{u}}=\sigma^{k_{0}+1}\left(\underline{\mathrm{u}}^{\prime}\right),
$$

and hence $\sigma^{k_{1}-k_{0}-1}(\underline{\mathrm{u}})=\underline{\mathrm{u}}$. Thus we have seen that $\underline{\mathrm{u}}$ is periodic, as claimed.

We now complete the proof of Theorem 1.2 by covering the case where the singular value is escaping but not an endpoint. (Note that there are parameters that satisfy the hypotheses of both Theorem 4.3 and Theorem 4.4.)

4.4. Theorem. Suppose that $f(z)=\exp (z)+a$ is an exponential map with $a \in I(f)$ such that $a$ is a non-endpoint. Then $I(f)$ is connected.

Proof. The singular value is accessible by Proposition 2.1. As shown in the proof of Theorem 4.3, if $I(f)$ was disconnected, there would be an unbounded, closed, connected set $A \subset \mathbf{C} \backslash I(f)$ and some number $k_{0}$ such that $f^{k_{0}+1}(A) \cup\{a\}$ is closed and connected. But this is impossible by Proposition 2.6.

\section{References}

[B] BARAŃsKi, K.: Trees and hairs for some hyperbolic entire maps of finite order. - Math. Z. $257: 1,2007,33-59$.

[DG] Devaney, R. L., and L. R. GoldBerG: Uniformization of attracting basins for exponential maps. - Duke Math. J. 55:2, 1987, 253-266.

[DGH] Devaney, R. L., L. R. Goldberg, and J.H. Hubbard: A dynamical approximation to the exponential map by polynomials. - Preprint, MSRI Berkeley, 1986.

[DK] Devaney, R. L., and M. Krych: Dynamics of $\exp (z)$. - Ergodic Theory Dynam. Systems $4: 1,1984,35-52$.

[DT] Devaney, R. L., and F. Tangerman: Dynamics of entire functions near the essential singularity. - Ergodic Theory Dynam. Systems 6:4, 1986, 489-503.

[E] Eremenko, A. Ė.: On the iteration of entire functions. - In: Dynamical systems and ergodic theory (Warsaw, 1986), Banach Center Publ. 23, PWN, Warsaw, 1989, 339-345.

[EL] Eremenko, A. È., and M. Yu. Lyubich: Dynamical properties of some classes of entire functions. - Ann. Inst. Fourier (Grenoble) 42:4, 1992, 989-1020.

[FRS] Förster, M., L. Rempe and D. Schleicher: Classification of escaping exponential maps. - Proc. Amer. Math. Soc. 136:2, 2008, 651-663.

[J] Jarque, X.: On the connectivity of the escaping set for complex exponential Misiurewicz parameters. - Proc. Amer. Math. Soc. (to appear).

[M-B] Mihaljević-Brandt, H.: Semiconjugacies, pinched cantor bouquets and hyperbolic orbifolds. - Trans. Amer. Math. Soc. (to appear), arXiv:0907.5398.

[R1] Rempe, L.: Topological dynamics of exponential maps on their escaping sets. - Ergodic Theory Dynam. Systems 26:6, 2006, 1939-1975.

[R2] Rempe, L.: On a question of Eremenko concerning escaping sets of entire functions. - Bull. London Math. Soc. 39:4, 2007, 661-666. 
[R3] Rempe, L.: On nonlanding dynamic rays of exponential maps. - Ann. Acad. Sci. Fenn. Math. 32, 2007, 353-369.

[R4] Rempe, L.: Rigidity of escaping dynamics for transcendental entire functions. - Acta Math. 203:2, 2009, 235-267.

[R5] Rempe, L.: The escaping set of the exponential. - Ergodic Theory Dynam. Systems 30, 2010, 505-599.

[ReS] Rempe, L., and D. Schleicher: Bifurcation loci of exponential maps and quadratic polynomials: local connectivity, triviality of fibers, and density of hyperbolicity. - In: Holomorphic dynamics and renormalization, Fields Inst. Commun. 53, Amer. Math. Soc., Providence, RI, 2008, 177-196.

[RS1] Rippon, P. J., and G. M. Stallard: On questions of Fatou and Eremenko. - Proc. Amer. Math. Soc. 133:4, 2005, 1119-1126.

[RS2] Rippon, P. J., and G. M. Stallard: Escaping points of entire functions of small growth. - Math. Z. 261:3, 2009, 557-570.

$\left[\mathrm{R}^{3} \mathrm{~S}\right]$ Rottenfusser, G., J. RÜCKert, L. Rempe, and D. Schleicher: Dynamic rays of entire functions. - Ann. of Math. (2) (to appear).

[SZ] Schleicher, D., and J. Zimmer: Escaping points of exponential maps. - J. London Math. Soc. (2) 67:2, 2003, 380-400.

Received 18 November 2009 